\title{
Seedlings of two Acacia species from contrasting habitats show different photoprotective and antioxidative responses to drought and heatwaves
}

\author{
Agnieszka Wujeska-Klause • Gerd Bossinger • Michael Tausz
}

Received: 8 July 2014 / Accepted: 4 November 2014 /Published online: 19 November 2014

(C) INRA and Springer-Verlag France 2014

\begin{abstract}
- Key message Two Acacia species adapted to contrasting habitats showed different response of photoprotective and antioxidative defence systems to imposed drought and heatwave.

- Context Predicted increases in drought frequency and intense heatwaves are expected to lead to dieback of sensitive tree species. Stomatal closure restricts $\mathrm{CO}_{2}$ input into the leaf, resulting in imbalances between light energy-driven electron transport rate and electron consumption in the Calvin cycle. Reactive oxygen species formed under these circumstances have to be kept under control by photoprotective and antioxidative defence systems.

- Aims We hypothesised that these defence systems behave differently in tree species from contrasting habitats.

- Methods Acacia aneura (adapted to arid habitats) and Acacia melanoxylon (adapted to humid habitats) were exposed to two water treatments for 50 days including two short heatwave periods. Responses were assessed by gas exchange,
\end{abstract}

Handling Editor: Erwin Dreyer

Contribution of the co-authors Agnieszka Wujeska-Klause: designing and conducting the experiment, writing the manuscript and running data analysis

Gerd Bossinger: supervising the work, detailed reading and revision of the manuscript and coordinating the research project

Michael Tausz: supervising the work, detailed reading and revision of the manuscript and coordinating the research project

\footnotetext{
A. Wujeska-Klause $(\bowtie) \cdot G$. Bossinger $\cdot M$. Tausz

Department of Forest and Ecosystem Science, The University of

Melbourne, 4 Water Street, Creswick, Victoria 3363, Australia

e-mail: awujeska@student.unimelb.edu.au

G. Bossinger

e-mail: gerd@unimelb.edu.au

M. Tausz

e-mail: michael.tausz@unimelb.edu.au
}

chlorophyll fluorescence and concentrations of antioxidants (phyllodes, roots).

- Results Photosynthesis and quantum yield of photochemistry decreased significantly in both Acacia species, especially after water was withheld in combination with the second heatwave episode. In phyllodes, the concentration of antioxidants remained unchanged until exposure to severe drought and heatwave conditions (except for A. melanoxylon where changes in glutathione concentration were observed prior to exposure to severe stress), but after water was withheld and the second heatwave occurred, oxidised forms of glutathione increased. After exposure to the second heatwave, wellwatered seedlings of A. melanoxylon but not $A$. aneura increased ascorbic acid concentration in phyllodes. Under wellwatered conditions, Acacia species also showed increased concentration of antioxidants in roots following heatwaves.

- Conclusions Both Acacia species showed photodamage to photosystem II (PSII) after water was withheld and the second heatwave imposed, but with more gradual response in $A$. aneura. Total concentration of investigated antioxidants increased in response to the first (A. melanoxylon) and second ( $A$. aneura) heatwaves rather than drought stress alone.

Keywords Acacia aneura $\cdot$ Acacia melanoxylon - ASC . GSH $\cdot$ Arid $\cdot$ Humid $\cdot$ Phyllodes $\cdot$ Roots

\section{Introduction}

According to global warming predictions, droughts and heatwaves are expected to become more frequent and longer (IPCC 2013). Drought is already a common climate factor in many regions, but if drought periods increase in intensity or frequency along with more intense heatwaves, then tree species can be negatively affected (Hennessy et al. 2008; IPCC 2013; Teskey et al. 2014). Sensitive species may be unable to 
acclimate to extreme weather conditions, which can lead to their dieback with serious effects on environmental sustainability (Allen et al. 2010). Strategies of trees to cope with drought and heat periods may be different between species from arid habitats and species from humid habitats, and the effectiveness of coping strategies can depend on exposure time and stress intensity (McDowell et al. 2008; O'Grady et al. 2009). Decrease of rainfall and more intense heatwaves are likely to cause decline among those tree species adapted to more humid habitats, whereas tree species adapted to arid environments may be more tolerant (Kubiske and Abrams 1994; McDowell et al. 2008). In addition, trees at the seedling stage may be more affected than adult trees, with significant repercussions for the regeneration of a species (Johnson et al. 2011; Teskey et al. 2014).

Environmental stress in general, and drought or heat more specifically, commonly causes photooxidative stress in photosynthetic tissues, a situation where an imbalance between absorbed light energy and energy consumption in the photosynthetic apparatus leads to increased formation of potentially harmful reactive oxygen species (Smirnoff 1993; Asada 2006). In the case of drought stress, stomatal closure is a common early response of plants to limit transpiration (Smirnoff 1993; Chaves et al. 2009; Teskey et al. 2014). Stomatal closure also restricts $\mathrm{CO}_{2}$ influx into the leaf, which reduces the consumption and regeneration of metabolic energy carriers (NADPH, ATP) in carbon fixation (Smirnoff 1993; Chaves et al. 2009). Despite flexible downregulation of photosystem II (PSII) efficiency, the electron transport chain can continue to transport electrons at higher rates than can be used for the production of metabolic energy carriers (Tausz et al. 2004; Asada 2006; Foyer et al. 2012). As a result, reaction centres of photosystems remain closed (they are unable to accept another electron) and excitation energy or electrons can be transferred to other compounds (foremost among them molecular oxygen), leading to increased generation of reactive oxygen species (ROS) (Tausz et al. 2004; Asada 2006; Kim and Apel 2013).

Plants possess several defence systems to avoid and control the formation of ROS in the photosynthetic apparatus. As a 'first line of defence', the quantum efficiency of electron flow is continuously and flexibly adjusted through processes regulated by thylakoid $\mathrm{pH}$ and involving protective xanthophylls that mediate dissipation of absorbed light energy as heat (Demmig-Adams et al. 2012). Other mechanisms, such as photorespiration, cyclic electron flow and the Mehler reaction and heat dissipation, serve as alternative electron sinks maintaining controlled electron flow when carbon assimilation is limited (Asada 2006; Foyer et al. 2012; Noctor et al. 2014). These processes can prevent the production of singlet oxygen and help avoid photoinhibition (Kim and Apel 2013; Noctor et al. 2014), but they are also a source of $\operatorname{ROS}\left(\mathrm{H}_{2} \mathrm{O}_{2}, \mathrm{O}_{2}{ }^{-}\right)$that need to be kept under control.
Production of ROS occurs to some extent also under nonstress conditions, but their concentration is kept under control by the antioxidative defence system (Noctor et al. 2014). An elevated concentration of ROS can be harmful for cell membranes (proteins, lipids) and nucleic acids due to the reactive nature of ROS (Smirnoff 1993; Foyer and Noctor 2009). Nonenzymatic antioxidants, such as the ubiquitous low molecular weight compounds ascorbic acid and glutathione, are an important part of plant defence systems. They are also linked to the activity of enzymatic antioxidants and together form a complex network of defence and redox signalling systems (i.e. ascorbate-glutathione cycle) (Smirnoff 1993; MunnéBosch 2005; Noctor et al. 2014). Ascorbic acid is abundant in plant cells and has important roles in ROS scavenging and in the regeneration of other antioxidants ( $\alpha$-tocopherol or xanthophylls), as well as in the regulation of cell division and elongation (Potters et al. 2002; Foyer and Noctor 2009). Glutathione is a low molecular weight compound with important roles as a ROS scavenger, in the regeneration of oxidised ascorbic acid (glutathione-ascorbate cycle) and in sulphur assimilation (Potters et al. 2002; Noctor et al. 2012). Notwithstanding the importance of many other antioxidative compounds in plant tissues (for example, $\alpha$-tocopherol, carotenes, xanthophylls, phenolics), this study focused on the central redox regulators ascorbic acid and glutathione (Foyer and Noctor 2011).

Antioxidants also play an important role in the growth and development of fine roots, where antioxidants can be synthetised or supplied from the canopy (Herschbach et al. 2009). Since the root system is exposed directly to decreasing soil water content (Chaves et al. 2009), changes in the concentration of antioxidants may indicate adjustments of the root system to changing growth conditions, such as low soil moisture conditions or elevated temperature (Shvaleva et al. 2005). Although not many studies described the role of antioxidants in defence systems of roots upon exposure to stress conditions, some authors showed an increase in the concentration of ascorbic acid and $\alpha$-tocopherol in response to low soil water content, whereas glutathione concentrations did not change (Haberer et al. 2008).

Response to stress depends on its duration and intensity, age of plants or adaptation to their habitat (Smirnoff 1993; Rahantaniaina et al. 2013). Reduced forms of antioxidants react with ROS, leading to their transformation into oxidised forms (Foyer and Noctor 2011). Increasing concentrations of the reduced forms of antioxidants upon stress exposure can indicate an active defence response of plants and mark the ability to withstand stress. Elevated concentrations of oxidised forms may indicate that the defence capacity is overwhelmed (Foyer and Noctor 2011; Rahantaniaina et al. 2013). In addition, initial small changes of the redox state (ratio of oxidised to reduced form) of ascorbic acid or glutathione may also be triggers for subsequent metabolic responses and therefore part 
of the stress signalling process (Tausz et al. 2004). Most studies seem to suggest that the concentration of nonenzymatic antioxidants increases under initial stages of stress, but progressing stress can lead to decreased concentrations and therefore reduced detoxification capacity, which is in part dependent on the concentration of reduced antioxidants (Smirnoff 1993; Tausz et al. 2004; Wujeska et al. 2013; Noctor et al. 2014). A recent metaanalysis by Wujeska et al. (2013) suggested that tree species from different ecosystems and with putatively different stress tolerance showed differences in their antioxidative responses to drought stress. Because across different species there are potentially many confounding effects, it was proposed in that study to investigate antioxidative defence system responses between ecologically contrasting, but genetically closely related, species (such as species from the same genus).

Australian native tree species offer such an opportunity, as a large proportion of all species belong to the genera Eucalyptus and Acacia. For instance, species of the genus Acacia are widely distributed in Australia, often closely related to each other, and can alternate along rainfall gradients.

To test whether antioxidative responses to stress are different between congenital species from contrasting habitats, we tested two Acacia species from contrasting habitats: A. aneura F. Muell ex Benth is adapted to arid habitats and A. melanoxylon $\mathrm{R}$. Br. to humid habitats. A. melanoxylon is a fast-growing and widely occurring tree species in highrainfall areas of Victoria, New South Wales and Queensland (Costermans 2006), whereas A. aneura is a slow-growing tree with a shallow root system and distributed in low-rainfall regions of Central Australia (O'Grady et al. 2009). We hypothesised that $A$. aneura, adapted to arid conditions, will be better equipped to cope with prolonged drought and heatwaves compared to A. melanoxylon. The strategy of $A$. aneura may include (1) stomatal responses consistent with anisohydric behaviour (e.g. O'Grady et al. 2009), (2) successful avoidance of photodamage and photooxidative stress, (3) no or smaller changes in the concentration of antioxidants (Wujeska et al. 2013) and (4) increased concentration of antioxidants in roots in response to drought stress and high temperature.

\section{Materials and methods}

\subsection{Plant material and growth conditions}

Two Acacia species from environments contrasting in their aridity were chosen: $A$. aneura F. Muell ex Benth adapted to areas with lower rainfall (annual rainfall $200-400 \mathrm{~mm}$, exact provenance unknown) and A. melanoxylon $\mathrm{R}$. Br. to higher rainfall (annual rainfall $750-1500 \mathrm{~mm}$; provenance
Smithton, Tasmania) (SpeciesBank 2014). Eight-month-old seedlings were bought from Meredith Nursery (Meredith, Victoria, Australia), repotted in $1.5-\mathrm{L}$ pots and grown for 5 months in a semi-controlled glasshouse (Creswick Campus of the University of Melbourne, $143^{\circ} 53^{\prime \prime} \mathrm{E}$, $37^{\circ} 25^{\prime \prime} \mathrm{S}$ ). Three units of potting mix for native species including slow-release fertiliser (Native Mix Superior Potting \& Planting Mix, Debco, Australia) were mixed with two units of coarse-grade sand (Propagating sand, Brunnings, Australia). For each species, 80 plants were grown, which were then randomly assigned to one of two treatments: well-watered and drought-stressed. For repeated gas exchange and chlorophyll fluorescence measurements, five plants per treatment were used repeatedly each day during the 50 days of the experiment. The remaining plants (35 per treatment) were used for destructive sampling and biochemical analysis (antioxidants). Plants of both drought-stressed and well-watered treatments were positioned randomly and interspersed with each other.

\subsection{Water treatments and glasshouse conditions}

Before starting the drought treatment, all seedlings were watered with the same amount of water (kept at or close to field capacity - FC). Plants were watered to FC and weighed (with soil and pot) to determine water use. During the experiment, seedlings were watered and weighed every $2-4$ days, depending on the species requirements (A. melanoxylon received more water more often than $A$. aneura). From day 1 to day 28 of the treatment, plants in the drought-stressed treatment group received $50 \%$ of the water used by well-watered plants. After day 28, drought-stressed plants were left without watering until day 50. The percentages of FC (maximum soil water content (SWC) calculated after watering) and minimum soil water content (min. SWC, calculated from the pot weight recorded immediately before watering) were calculated based on gravimetric measurements through this time.

Plants received natural light in the glasshouse (approx. 70$80 \%$ of outdoor photosynthetic photon flux density (PPFD)), and the glasshouse was tracking outside conditions except for a cooling system, which was set to maintain day temperatures below a maximum of $25^{\circ} \mathrm{C}$. Technical problems with the cooling system from day 28 to day 30 coincided with sunny conditions outdoors exposing all plants to a simulated heatwave, and to a lesser extent also at the beginning of the experiment from day 3 to day 5 . Air temperatures during these periods exceeded $30{ }^{\circ} \mathrm{C}$. Spot measurements using the leaf thermocouple of a LI-COR 6400 (LI-COR, Lincoln, NE, USA) indicated that phyllode temperatures reached well above $30{ }^{\circ} \mathrm{C}$ between day 28 and day 30 , whereas they remained below $30{ }^{\circ} \mathrm{C}$ at all other times. 


\subsection{Gas exchange and chlorophyll fluorescence}

Gas exchange and chlorophyll fluorescence were measured with a LI-COR 6400 (leaf fluorometer chamber 6400-40) on the same phyllodes and seedlings between 10.00 a.m. and 1.00 p.m. On the same day as gas exchange measurements, pre-dawn chlorophyll fluorescence measurements were performed using a portable fluorometer $\left(\mathrm{OS} 30 \mathrm{p}_{+}\right.$, Opti-Sciences, Inc., Hudson, NH, USA) and the maximum quantum efficiency of PSII $\left(F_{v} / F_{m}\right)$ was determined. For gas exchange and simultaneous fluorescence measurements, LI-COR conditions were as follows: $\mathrm{CO}_{2}$ concentration (reference) was set at $400 \mathrm{ppm}$, flow rate at $500 \mu \mathrm{mol} \mathrm{m} \mathrm{s}^{-1}$ (A. melanoxylon) or $250 \mu \mathrm{mol} \mathrm{m}{ }^{-2} \mathrm{~s}^{-1}$ (A. aneura; to account for the smaller phyllodes) and light intensity at $1200 \mu \mathrm{mol} \mathrm{m} \mathrm{m}^{-2} \mathrm{~s}^{-1}$ PPFD (which was found to be saturating for both species). Phyllode temperature during measurements was $20-25^{\circ} \mathrm{C}$ and relative humidity in the chamber $50-60 \%$. The following parameters were recorded: net $\mathrm{CO}_{2}$ assimilation rate $(A)$, stomatal conductance $\left(g_{\mathrm{s}}\right)$ and efficiency of open reaction centres in PSII in the light $\left(F_{v}^{\prime} F_{m}\right)$. After 50 days, these plants were used for antioxidant analysis. Relative stomatal conductance (rel $g_{\mathrm{s}}$ ) was calculated by dividing each $g_{\mathrm{s}}$ value by the maximum $g_{\mathrm{s}}$ obtained for the species and the whole experiment, determined as the 95-percentile of all $g_{\mathrm{s}}$ measurements. At the beginning of the experiment, mean leaf mass area (LMA) was 213.9 $63.7 \mathrm{~g} \mathrm{~m}^{-2}$ (A. melanoxylon) and $254.6 \pm 94.5 \mathrm{~g} \mathrm{~m}^{-2}$ (A. aneura) $(p=0.274)$.

\subsection{Glutathione and ascorbic acid determination}

Glutathione and ascorbic acid concentration was measured on fully expanded phyllodes and fine roots $(<2$-mm diameter). Samples were frozen in liquid nitrogen immediately and then freeze-dried for $72 \mathrm{~h}$. Afterwards, samples were weighed (ca. $50-60 \mathrm{mg}$ of plant material) and ground to a fine powder with a pestle, a mortar, equal amounts of polyvinylpolypyrrolidone (PVPP), fine quartz sand and liquid nitrogen. Then, $1.5 \mathrm{~mL}$ of $4.5 \%(w / v)$ metaphosphoric acid was added and mixed by vortex. The samples were centrifuged for $2.5 \mathrm{~min}$ at $16,100 \times \mathrm{g}$. The supernatants (extracts) were decanted and stored at $-20^{\circ} \mathrm{C}$ for later analysis.

A 5,5'-dithio-bis-(2-nitrobenzoic acid) (DTNB) assay was used to determine the total (GSH+GSSG) and oxidised glutathione (GSSG). Aliquots of the extracts were neutralised in potassium phosphate buffer $(250 \mathrm{mM}, \mathrm{pH} 7)$, and to one of the aliquots, 2-vinylpyridine was added to block the reduced form of glutathione and assess GSSG concentration. Excess 2vinylpyridine was removed by adding triethanolamine to the extract. The other aliquot of the extract did not receive 2vinylpyridine and was used to determine the total (GSH+ GSSG). A working solution containing DTNB, dimethyl sulphoxide (DMSO) and glutathione reductase was mixed with the extracts and $\beta$-nicotinamide adenine dinucleotide phosphate reduced (NADPH) was used to start the reaction. Glutathione concentrations of both forms were determined by recording absorbance at $412 \mathrm{~nm}$ at room temperature. Concentrations of the total and oxidised form were calculated according to Sgherri et al. (1994). Reduced GSH was assessed by the difference between total GSH and GSSG. This method is based on Knörzer et al. (1996).

To determine total ascorbic acid (ASC+dehydroascorbic acid (DHA)), the supernatant (extract) was neutralised in sodium phosphate buffer $(150 \mathrm{mM}, \mathrm{pH}$ 7.4) containing triethanolamine $(1.5 \mathrm{M} \mathrm{v} / v)$, and then, DL-Dithiothreitol (DTT, $20 \mathrm{mM}$ ) was used to reduce DHA to ASC. After incubation for $15 \mathrm{~min}, \mathrm{~N}$-ethylmaleimide (NEM, $0.5 \%(w /$ $v)$ ) was added to remove excess DTT from the extract. To determine the reduced form of ASC, replicate extracts were treated with de-ionised water. Ascorbic acid in the extracts was determined by treating with trichloroacetic acid $(10 \% \mathrm{w} /$ $v)$, orthophosphoric acid $(40 \% v / v), 2,2^{\prime}$-dipyridil $(4 \%$ in $70 \%$ ethanol) and iron(III) chloride $(15 \% \mathrm{w} / \mathrm{v})$. After incubation for $1 \mathrm{~h}$ at $37^{\circ} \mathrm{C}$, the concentration of total and reduced ascorbic acid was determined using absorbance at $525 \mathrm{~nm}$ at room temperature. This method is based on Knörzer et al. (1996).

\subsection{Statistical analysis}

Statistical analysis and graphs were performed using RStudio (version 0.98.501; open source software; (C) 2009-2013 RStudio, Inc.). For the analysis of repeated measurements (gas exchange and chlorophyll fluorescence) on the same individual seedlings for the entire 50 days, a linear mixed effects model was used ('Ime' from R package 'nmle'). Day of the experiment was used as the within-subjects factor, and species and treatment were the between-subjects factors. Antioxidants at each sampling day were analysed using a univariate general linear model with the factors day, treatment (well-watered and drought-stressed) and species (A. aneura and $A$. melanoxylon) ('aov' from $\mathrm{R}$ package 'stats'). Individual differences between well-watered and droughtstressed plants on each experimental day were determined using $t$ test ('t.test' from $\mathrm{R}$ package 'stats'). For all analyses, $p$ values of less than 0.05 were considered statistically significant $\left({ }^{*} p<0.05 ;{ }^{* *} p<0.01 ; * * * p<0.001\right)$. In addition, individual differences between the species for each treatment separately and at each sampling day were determined using $t$ tests ('t.test' from R package 'stats'). To determine if data deviated from normality, Levene's test was used, and when the test was significant ('leveneTest' from R package 'car'), $\log$ data transformations were used. Data were checked visually for the correlations between variances and means. To determine the relationships of stomatal responses to the percentage of FC, the three-parameter sigmoid curve was used 
and calculated using the equation: $g_{\mathrm{s}}=\frac{a}{1+e^{-\frac{\mathrm{FFC}-c}{b}}}$ (Brodribb and Cochard 2008). The curve was fitted by using quantile regression to estimate the boundary line at the $95 \%$ level for each species separately. Curves were constrained to 0 at low soil water content and rel $g_{\mathrm{s}}$ to reflect the distribution of the points. Quantile regression was performed with the quantreg package (version 5.05) (Koenker et al. 2013).

\section{Results}

\subsection{Gas exchange and chlorophyll fluorescence}

Drought stress reduced net $\mathrm{CO}_{2}$ assimilation rate ( $A$; Fig. 1) and stomatal conductance ( $g_{\mathrm{s}}$; Fig. 2) in both Acacia species. In $A$. aneura, $A$ and $g_{\mathrm{s}}$ decreased gradually, whereas they were more variable before declining sharply on the 28th day in A. melanoxylon (when high air temperature coincided with the withholding of water). Stressed seedlings showed a significant reduction of $A$ from day 9 and day 4, whereas $g_{\text {s }}$ decreased significantly from day 9 and day 7 (A. aneura and A. melanoxylon, respectively). There were no significant changes in well-watered seedlings of both species throughout the whole experiment. Soil moisture decreased gradually for drought-stressed seedlings and remained constant in wellwatered ones for both Acacia species (Fig. 3). In A. aneura, relative stomatal conductance ( $\mathrm{rel} g_{\mathrm{s}}$ ) was 1 when soil moisture ranged between 100 and $60 \%$ FC (Fig. 4). In contrast, in A. melanoxylon, rel $g_{\mathrm{s}}$ decreased to $\sim 0.8$ at $75 \%$ of FC. When soil moisture reached $50 \% \mathrm{FC}$, rel $g_{\mathrm{s}}$ of $A$. aneura still was relatively high ( $\mathrm{rel} g_{\mathrm{s}}=\sim 0.9$ ), whereas in $A$. melanoxylon, it dropped below 0.5 of rel $g_{\mathrm{s}}$. Both species had rel $g_{\mathrm{s}}$ close to 0 when soil moisture dropped below $10 \%$ FC. Minimum soil water content ( $\mathrm{min}$. SWC in \%) was constant for well-watered seedlings in pots of both Acacia species during the experiment (except for a drop on day 11 in pots of both treatments of $A$. melanoxylon) (Fig. 3). Min. SWC was decreasing gradually in pots of drought-stressed seedlings (both species), reaching around $10 \%$ at the end of the experiment. Min. SWC were comparable among species and ranged between 61 and $83 \%$ for wellwatered $A$. aneura, 10-61 \% for drought-stressed A. aneura, 46-88 \% for well-watered A. melanoxylon and 16-60\% for drought-stressed A. melanoxylon.

Maximum quantum efficiency of PSII in the dark $\left(F_{v} / F_{m}\right)$ and in the light $\left(F_{v} / F_{m}\right)$ decreased in drought-stressed seedlings after day 28 (when water was withheld in coincidence with high temperatures), whereas it remained high in wellwatered seedlings (Fig. 5). $F_{v} / F_{m}$ did not vary between treatments in both species until the 28th day. $F_{v}{ }^{\prime} / F_{m}{ }^{\prime}$ was reduced in drought-stressed A. melanoxylon seedlings on day 14 and day 21. It decreased significantly in drought-stressed trees of both species after day 28 .

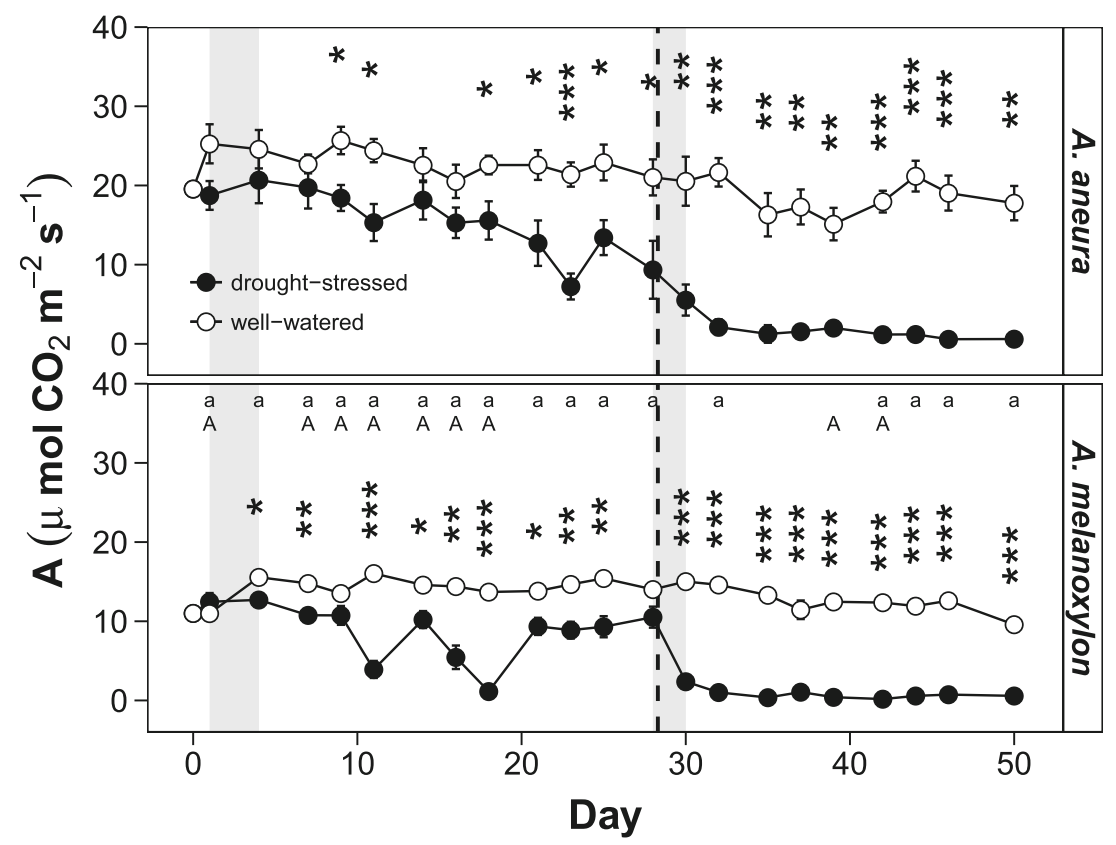

Fig. 1 Net $\mathrm{CO}_{2}$ assimilation rate $(A)$ of two Acacia species (A. aneura and A. melanoxylon) under well-watered (open circle) and droughtstressed (closed circle) treatments. The vertical line indicates the start of a period where water was withheld completely. The shaded areas indicate the time of heatwaves when air temperatures exceeded $30{ }^{\circ} \mathrm{C}$. Values are means $( \pm \mathrm{SE})$ of $n=5$. Effects of sampling day, species and treatment as well as their interaction were statistically significant $(p<0.01)$, and asterisks indicate significant differences between water treatments at each sample time $(* p<0.05 ; * * p<0.01 ; * * * p<0.001)$. Letters indicate significant differences between the species under drought-stressed (capital letters) and well-watered treatment (small letters) 
Fig. 2 Stomatal conductance $\left(g_{\mathrm{s}}\right)$ of two Acacia species (A. aneura and A. melanoxylon) under wellwatered (open circle) and drought-stressed (closed circle) treatments. Vertical line, shaded areas, letters and asterisks described as in Fig. 1. Values are means ( \pm SE) of $n=5$. Effects on all levels and their interactions were statistically significant $(p<0.001)$

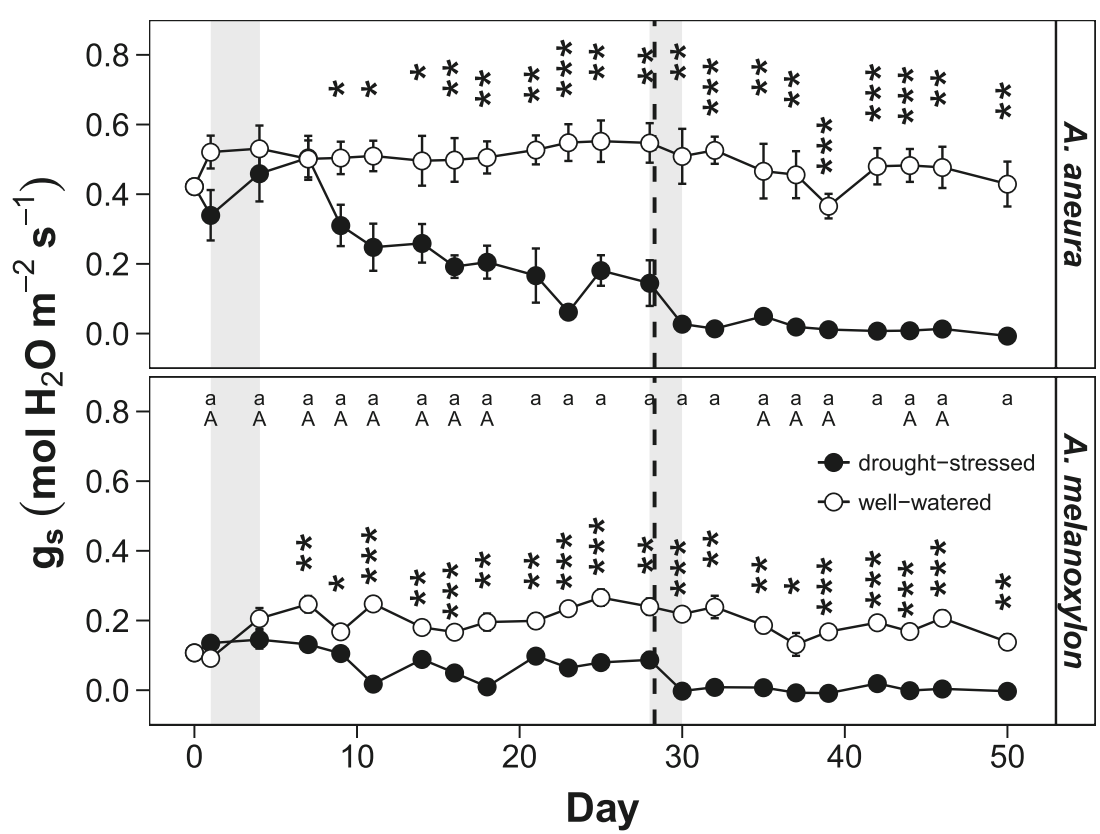

3.2 Concentration of non-enzymatic antioxidants in phyllodes and roots

\subsubsection{Glutathione}

In A. melanoxylon, total glutathione concentration (GSH+ GSSG; Fig. 6) was lower in phyllodes of drought-stressed seedlings than in well-watered ones (already significant on day 14), whereas total glutathione in $A$. aneura did not change between treatments until the last day. In A. melanoxylon, GSH+GSSG did not show an increase in the concentration after day 28 (when trees were exposed to heatwave conditions). Phyllode GSH+
GSSG seemed to respond to some extent to heat events with more rapid response after the first heatwave. Both species showed a peak in phyllode GSH+ GSSG concentration following the earlier heat period, and $A$. aneura but not $A$. melanoxylon also showed such a peak a few days after the second heatwave, irrespective of drought-stressed treatments (Fig. 6). The ratio of oxidised to total glutathione (GSSG\%; Fig. 6) increased in drought-stressed seedlings of both Acacia species after day 28. A. melanoxylon seedlings also showed a peak of $\mathrm{GSSG} \%$ during the first heat period, and also well-watered seedlings of A. melanoxylon (but not $A$. aneura) showed increased proportion of GSSG after the second heatwave.
Fig. 3 Minimum (dashed line) and maximum soil water contents (solid line, SWC) expressed as percentage of FC in pots of two Acacia species (A. aneura and A. melanoxylon) under wellwatered (open circle) and drought-stressed (closed circle) treatments. Vertical line and shaded areas described as in Fig. 1. Values are means $( \pm \mathrm{SE})$ of $n=5$. min. $S W C$ : Effects of sampling day and treatment as well as their interaction (species by treatment, treatment by day) were statistically significant $(p<0.01)$. max. SWC: Effects of sampling day, species and treatment as well as interactions (treatment by day) were statistically significant $(p<0.001)$

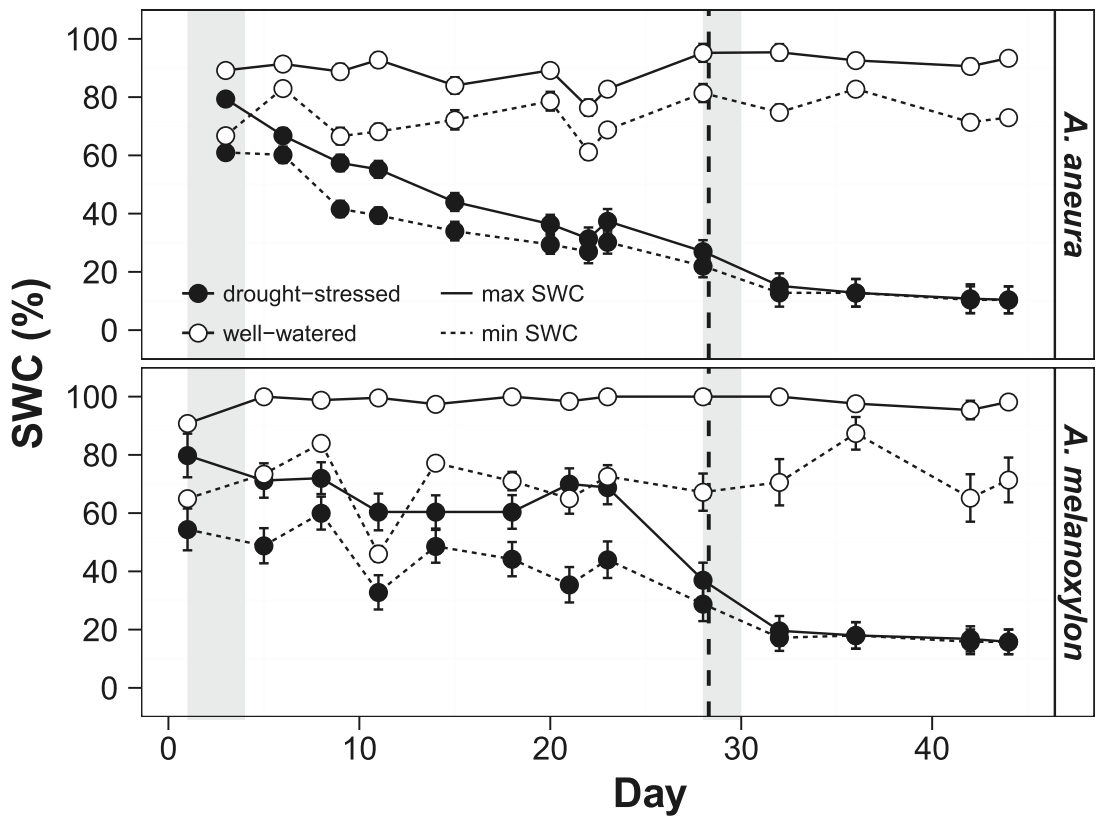




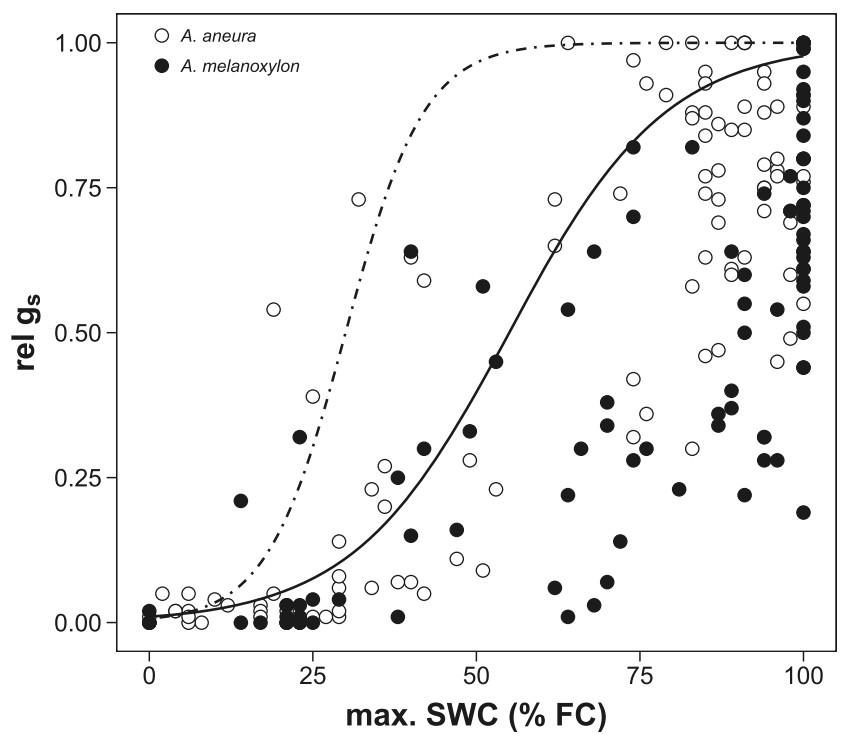

Fig. 4 Response of stomatal conductance of $A$. aneura (dot-dashed line; open circle) and A. melanoxylon (solid line; closed circle) expressed as relative stomatal conductance (rel $g_{\mathrm{s}}$ ) under decreasing maximum soil water content (max. SWC expressed in \%FC). Boundary lines $(95 \%)$ were plotted using three sigmoid function rel $g_{\mathrm{s}}=a /(1+\mathrm{e}(-(\% \mathrm{FC}-c) / b))$ for each species separately

The concentration of GSH + GSSG in roots was not significantly different between the water treatments in A. melanoxylon during the whole experiment (Fig. 7). In line with phyllode glutathione concentrations, root GSH+GSSG concentrations of well-watered and drought-stressed A. melanoxylon and well-watered A. aneura peaked around day 40, after the second heatwave. This increase was not observed in drought-stressed $A$. aneura, leading to a statistically significant difference between water treatments for this species on day 42 . The proportion of oxidised glutathione did not vary significantly between treatments of both Acacia species, except for a marginal but significant increase in A. melanoxylon at day 50 (Fig. 7). The GSSG\% increased slightly after the 28 th day in both species and treatments. This species also showed an increase in GSSG\% following the first heat period, similar to observations in phyllodes.

\subsubsection{Ascorbic acid}

Total phyllode ascorbic acid (ASC+DHA; Fig. 8) became significantly lower in drought-stressed than well-watered seedlings during later stages of the experiment. In A. aneura, this difference was only significant at the last day, whereas in A. melanoxylon, differences were more pronounced and became apparent from day 28 onwards. In A. melanoxylon, phyllode ASC +DHA concentrations of well-watered but not drought-stressed seedlings increased after the heatwave following day 28 , which resulted in the difference between water treatments. Ascorbic acid (ASC\%; Fig. 8) was in a highly reduced state (ca. $80 \%$ ) in both species throughout the

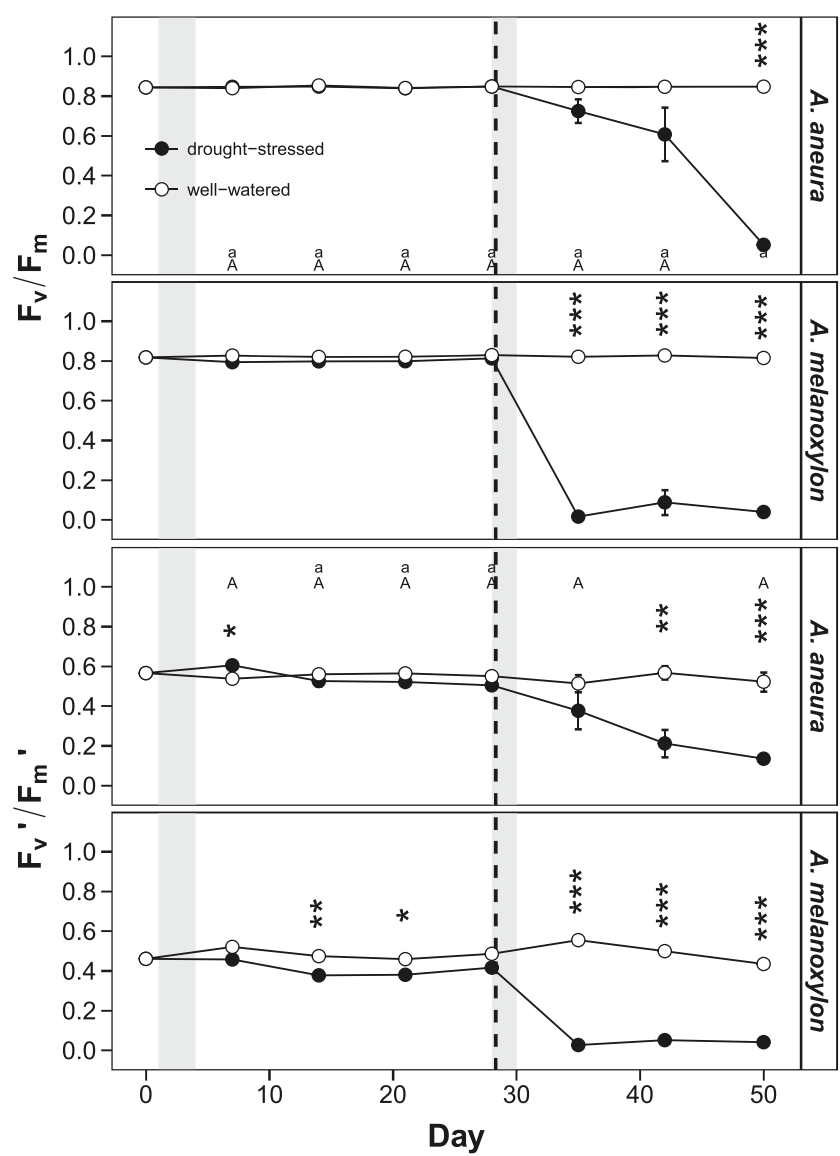

Fig. 5 Maximum quantum efficiency of PSII in the dark $\left(F_{v} / F_{m}\right)$ and maximum quantum efficiency of PSII in the light $\left(F_{v}{ }^{\prime} / F_{m}\right)$ of two Acacia species ( $A$. aneura and A. melanoxylon) under well-watered (open circle) and drought-stressed (closed circle) treatments. Vertical line, shaded areas, letters and asterisks described as in Fig. 1. Values are means ( $\pm \mathrm{SE}$ ) of $n=5 . F_{v} / F_{m}$ : Effects of sampling day, species and treatment as well as their interactions were statistically significant $(p<0.01) . F_{v}{ }^{\prime} / F_{m}{ }^{\prime}$ : Effects of sampling day, species and treatment as well as their interactions (species by treatment, treatment by day) were statistically significant $(p<0.01)$

experiment, but even though changes were small, the ascorbate pool in drought-stressed seedlings was slightly, but significantly, more oxidised towards the end of the experiment. In $A$. aneura, this became only evident on day 50, whereas in $A$. melanoxylon, this oxidation followed shortly after day 28 .

The concentration of ASC + DHA in roots did not change between treatments for $A$. aneura, except for very small, but significant, decreases in drought-stressed seedlings on day 42 and day 50 (Fig. 9). In A. melanoxylon, the difference between treatments was significant on day 42 . There was a significant increase in the ASC+DHA concentration in well-watered A. melanoxylon on day 14. Ascorbic acid (ASC\%; Fig. 9) was in a highly reduced state (80 \%) in roots of both species and in both treatments during the first week of the drought experiment. Roots of drought-stressed seedlings of both species showed an increase in the proportion of reduced ascorbate 


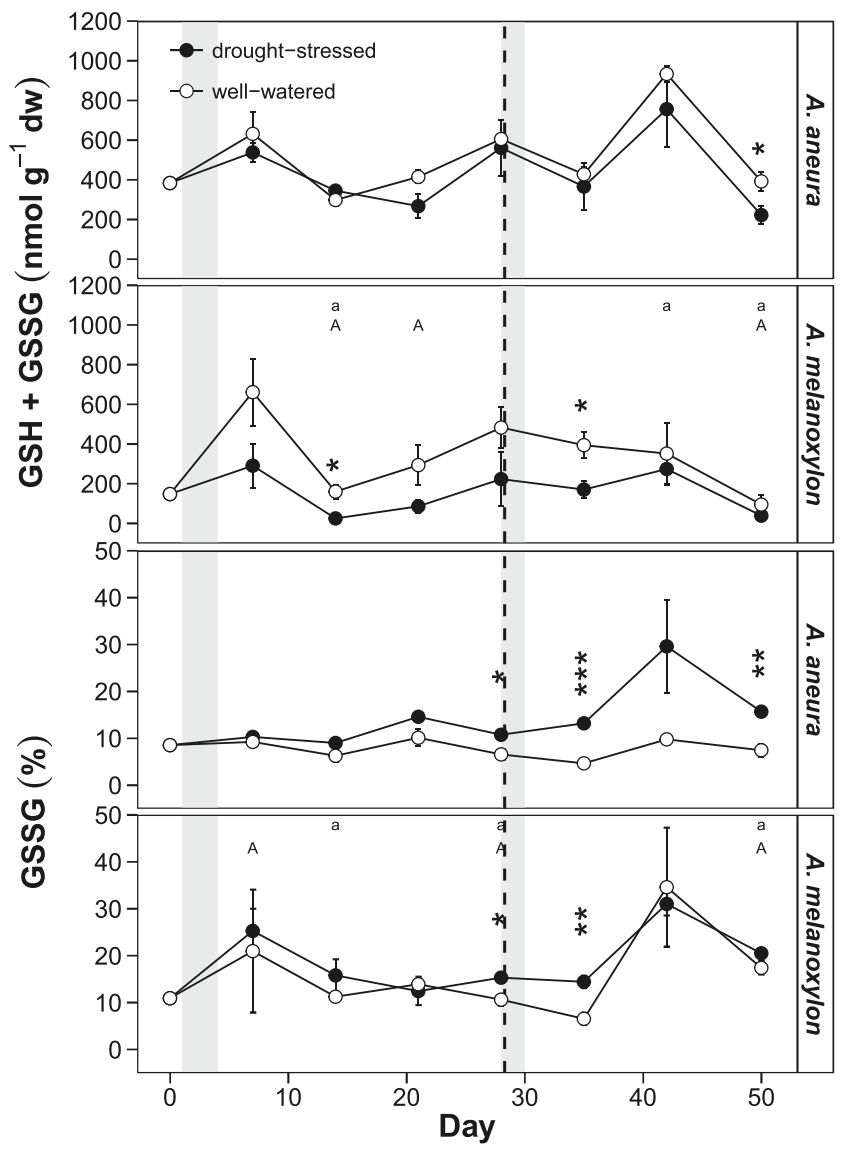

Fig. 6 Total glutathione concentration $(\mathrm{GSH}+\mathrm{GSSG})$ and ratio of oxidised to total glutathione (GSSG\%) in the phyllodes of two Acacia species (A. aneura and A. melanoxylon) under well-watered (open circle) and drought-stressed (closed circle) treatments. Vertical line, shaded areas, letters and asterisks described as in Fig. 1. Values are means $( \pm \mathrm{SE})$ of $n=5$. $G S H+G S S G$ : Effects of sampling day, species and treatment as well as their interaction (day by species) were statistically significant $(p<0.05)$. GSSG\%: Effects of sampling day, species and treatment were statistically significant $(p<0.01)$

(i.e. a more reduced, less oxidised state) during the final stages of the drought-stressed treatment.

\section{Discussion}

Stomatal closure is an important immediate response of plants exposed to drought stress. While reducing transpiration, stomatal closure also limits carbon assimilation and increases the risk of photooxidative stress (Smirnoff 1993; Chaves et al. 2009). In our experiment, the response of $g_{\mathrm{s}}$ to soil moisture was different between the investigated Acacia species. Stomata of $A$. aneura, the arid species, were fully open until $50 \%$ of FC which closed sharply upon further soil drying, whereas in A. melanoxylon, stomata were closing more gradually and already in response to moderate soil water deficits. A. aneura has been described as an anisohydric species, which

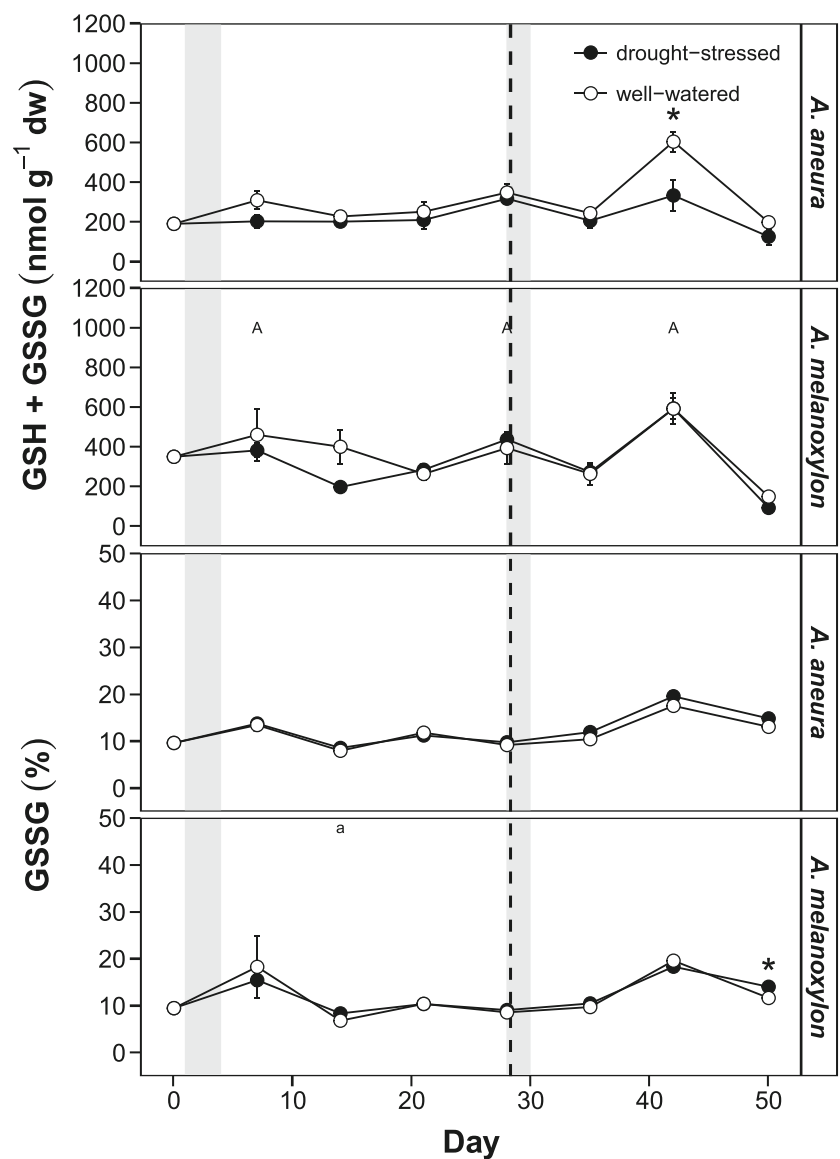

Fig. 7 Total glutathione concentration $(\mathrm{GSH}+\mathrm{GSSG})$ and ratio of oxidised to total glutathione (GSSG\%) in the roots of two Acacia species (A. aneura and A. melanoxylon) under well-watered (open circle) and drought-stressed (closed circle) treatments. Vertical line, shaded areas, letters and asterisks described as in Fig. 1. Values are means $( \pm \mathrm{SE})$ of $n=$ 5. GSH+GSSG: Effects of sampling day, species and treatment as well as their interaction (day by species) were statistically significant $(p<0.05)$. $G S S G \%$ : Effect of sampling day was statistically significant $(p<0.001)$

implies a capacity to tolerate or adjust to strongly negative phyllode water potentials (McDowell et al. 2008; O'Grady et al. 2009). The anisohydric behaviour of $A$. aneura may have allowed stomata to remain open at lower SWC, a strategy that has a positive effect on carbon balance, especially during prolonged drought of medium intensity, but can also bring a risk of hydraulic failure when drought stress becomes more intense (McDowell et al. 2008; O'Grady et al. 2009). This strategy is part of $A$. aneura adaptation to arid and semiarid habitats (O'Grady et al. 2009; ANBG 2010) and reflected in the differences in stomatal response compared to A. melanoxylon adapted to humid habitats.

Photosynthesis or more specifically Rubisco activity and regeneration of 1,5-bisphosphate (RuBP) can be negatively affected by high temperatures (Sharkey 2005; Rennenberg et al. 2006). At moderate temperatures, Rubisco can be reversibly deactivated while photorespiration is promoted as an alternative electron sink (Sharkey and Zhang 2010). Because 


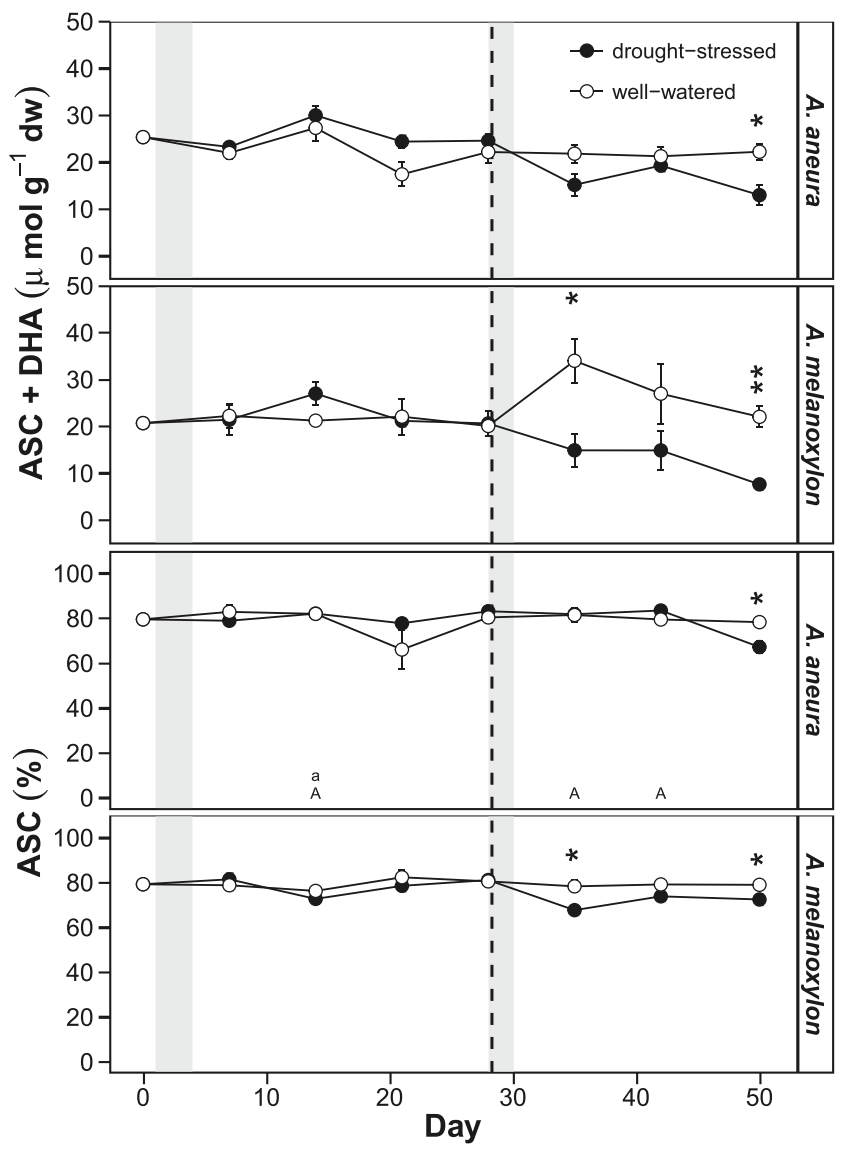

Fig. 8 Total ascorbic acid concentration (ASC+DHA) and ratio of reduced to total ascorbic acid (ASC\%) in phyllodes of two Acacia species (A. aneura and A. melanoxylon) under well-watered (open circle) and drought-stressed (closed circle) treatments. Vertical line, shaded areas, letters and asterisks described as in Fig. 1. Values are means $( \pm \mathrm{SE})$ of $n=$ 5. $A S C+D H A$ : Effects of sampling day, treatment as well as their interactions (day by treatment, species by treatment) were statistically significant $(p<0.05)$. ASC\%: Effects of sampling day as well as interactions were statistically significant $(p<0.05)$

well-watered trees of both Acacia species had constant $A$ and $g_{\mathrm{s}}$ throughout the experiment, it seems that the heatwave episodes alone did not have a strong effect on photosynthesis. When drought-stressed trees experienced a heatwave, $A$ and $g_{\mathrm{s}}$ responded strongly, suggesting that a combination of drought and heat elicited stronger responses than just decreasing water content (Teskey et al. 2014).

Upon stomatal closure, which also limits access of $\mathrm{CO}_{2}$ to the leaves and therefore carbon assimilation, plants need to efficiently regulate the dissipation of absorbed light energy as heat and use electrons in alternative pathways to avoid (photo)oxidative stress, processes that form 'first lines of defence' against oxidative stress (Demmig-Adams et al. 2012; Foyer et al. 2012; Noctor et al. 2014). The extent of heat dissipation can be inferred from the quantum efficiency of PSII as measured by chlorophyll fluorescence parameters (efficiency of open reaction centres in PSII in the light $\left(F_{v}{ }^{\prime} /\right.$ $F_{m}$ ) and maximum quantum efficiency of PSII $\left.\left(F_{v} / F_{m}\right)\right)$,

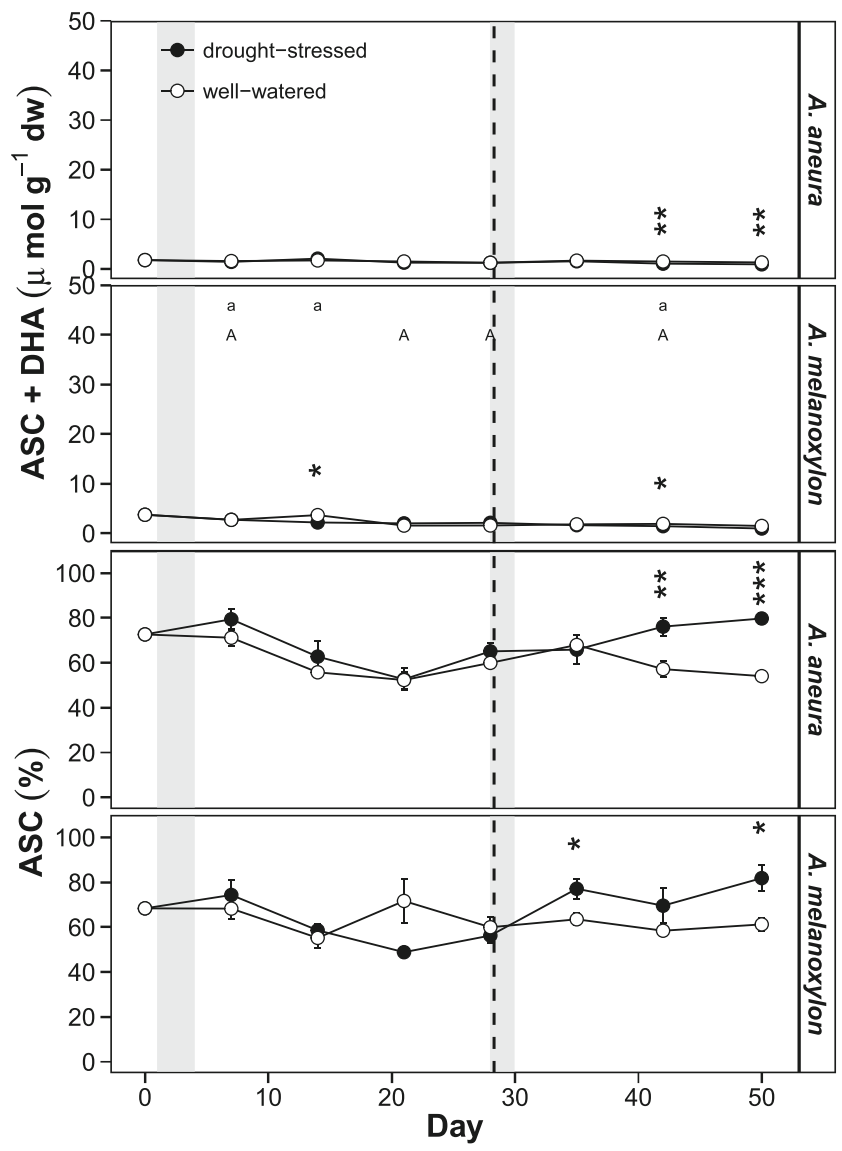

Fig. 9 Total ascorbic acid concentration (ASC+DHA) and ratio of reduced to total ascorbic acid (ASC\%) in roots of two Acacia species (A. aneura and A. melanoxylon) under well-watered (open circle) and drought-stressed (closed circle) treatments. Vertical line, shaded areas, letters and asterisks described as in Fig. 1. Values are means $( \pm \mathrm{SE})$ of $n=$ 5. ASC $+D H A$ : Effects of sampling day, species and treatment as well as their interactions (day by species, day by species by treatment, day by treatment) were statistically significant $(p<0.05)$. ASC\%: Effects of sampling day and treatment as well as their interaction were statistically significant $(p<0.001)$

which are especially useful under stress, i.e. drought or heat (Maxwell and Johnson 2000; Demmig-Adams et al. 2012). In our study, increased heat dissipation, which is associated with a reduction of the efficiency of open reaction centres in PSII in the light (Demmig-Adams et al. 2012), was indicated for A. melanoxylon already during early stages of the experiment. Maximum quantum efficiency of PSII values (i.e. the optimum quantum efficiency of PSII after a recovery phase), however, remained around 0.83 , which corresponds to undamaged PSII (Maxwell and Johnson 2000; DemmigAdams et al. 2012). This suggests that the engagement of a flexible and easily reversible heat dissipation mechanism was sufficient to avoid damages to PSII in A. melanoxylon under reduced water availability. A. aneura did not reduce either efficiency of open reaction centres in PSII in the light or maximum quantum efficiency of PSII during the earlier, deficit irrigation phase of the experiment. After water was 
withheld completely, and following a period of high temperatures in the glasshouse, efficiency of open reaction centres in PSII in the light decreased further along with a decrease in maximum quantum efficiency of PSII. Response of maximum quantum efficiency of PSII, indicative of photodamages to PSII (slowly reversible and requiring repair processes), was more gradual in A. aneura than in A. melanoxylon, where it developed immediately after watering was stopped and high temperatures occurred.

If adjustment of electron flow through flexible heat dissipation is insufficient to address potential imbalances between light-driven electrons and electron consumption in carbon fixation, the formation of ROS can increase (Asada 2006; Demmig-Adams et al. 2012; Foyer et al. 2012). Under relatively mild stress, ROS concentration can be regulated by the antioxidative defence system, but under more severe stress, the imbalance between ROS production and scavenging results in (photo)oxidative stress (Smirnoff 1993; Tausz et al. 2004; Noctor et al. 2014). It was suggested that responses of antioxidants to stress may depend on stress severity and stress progression: The concentration of non-enzymatic antioxidants can increase or be unresponsive at early or mild stages of drought while more severe stress can lead to the breakdown of the defence system with decreased antioxidant concentrations and increasingly oxidised antioxidant pools (Smirnoff 1993; Tausz et al. 2004; Wujeska et al. 2013; Noctor et al. 2014). In our study, concentrations of GSH+GSSG remained stable during early stages of the drought-stressed treatment in A. aneura phyllodes. However, progressing drought in combination with heat led to increased proportions of oxidised glutathione in a decreased total pool of glutathione in the phyllodes of drought-stressed $A$. aneura seedlings. A. melanoxylon showed similar changes in the concentration of GSH + GSSG but with an earlier and more rapid response following the first heatwave than A. aneura. These results are consistent with the notion that severe oxidative stress depletes the glutathione pool through oxidative processes (Smirnoff 1993; Tausz et al. 2004; Wujeska et al. 2013; Noctor et al. 2014). Ascorbic acid (ASC+DHA, ASC\%) showed a similar response to the treatments to glutathione. In both Acacia species, the concentration of ascorbic acid was unresponsive during early stages of the treatment, but severe drought in combination with heat led to lower ASC+DHA concentrations in phyllodes. This response was delayed for $A$. aneura in comparison to A. melanoxylon. Such a decrease, especially under severe stress, could be a result of a depleted ASC +DHA pool due to oxidative degradation (Smirnoff 1993; Tausz et al. 2004; Noctor et al. 2014). However, because the ascorbic acid pool remained in a highly reduced state (indicated by $\mathrm{ASC} \%$ ) normally associated with healthy tissues, the reduction of ASC + DHA in this experiment may also have been the result of an adjustment or regulation of the ascorbic acid pool, perhaps related to changes in synthesis or turnover rates of ascorbic acid (Foyer and Noctor 2011; Smirnoff 2011).
The response of the antioxidative defence systems coincided with chlorophyll fluorescence parameters indicating strong engagement of flexible heat dissipation (efficiency of open reaction centres in PSII in the light) and also damages to PSII (indicated by decreased maximum quantum efficiency of PSII). In agreement with results from an earlier meta-analysis addressing antioxidative responses of trees to drought (Wujeska et al. 2013), the responses of both ascorbic acid and glutathione in this present experiment seem to be dependent on stress intensity: Concentrations of antioxidants increase upon exposure to mild or moderate stress, but this is followed by breakdown of the foliar antioxidative defence system when stress became more severe.

In A. melanoxylon but not $A$. aneura, the heatwave exposure alone seemed to have a greater effect on the defence systems than drought, as even well-watered seedlings showed an oxidation of the glutathione pool after heatwaves. During the first week of the present experiment, the presence of juvenile leaves along with phyllodes in A. melanoxylon may have made drought-stressed seedlings more vulnerable especially to combined stress factors, which may explain the increased oxidation of glutathione in the absence of any putatively adaptive increase in GSH+GSSG (or ASC+ DHA) concentrations in deficitirrigated seedlings after the first heatwave. Well-watered seedlings indicated an acclimatory response to elevated temperature by increased ASC + DHA concentrations (Smirnoff 1993; Tausz et al. 2004). In contrast, $A$. aneura phyllodes seem to have a higher threshold for any such heat response, as indicated by the absence of any indication of oxidative stress and an increase of GSH + GSSG in well-watered seedlings during heat.

There are few investigations of ascorbic acid and glutathione in tree roots exposed to drought: A field study on European beech (Fagus sylvatica) concluded that root ascorbate concentrations increased in response to soil drying, but the response was overlaid by seasonal changes. Responses of root glutathione were seemingly unrelated to soil water content in that study (Haberer et al. 2008). In the present study, root glutathione concentrations of both Acacia species remained unchanged in response to drought treatments, but increased after heat in well-watered seedlings of $A$. aneura and irrespective of drought treatment in A. melanoxylon. The relationship of root antioxidative systems with environmental factors is not straightforward, as both ascorbic acid and glutathione in roots can be supplied from above-ground parts (Herschbach et al. 2009; Smirnoff 2011). In addition, both glutathione and ascorbic acid in roots are also associated with growth activities (Herschbach et al. 2009) and have been implied in whole-plant stress signalling, e.g. in relation to abscisic acid production (Zhao et al. 2005). It is possible that after the heatwave, roots responded with increased growth (or repair) activity (Herschbach et al. 2009), but it remains unclear why such a response was seen in glutathione but not in ascorbic acid. In one study, root glutathione responded to above-ground exposure to ozone (but not to soil drying), a 
result that can only be explained by shoot to root translocation of either glutathione or a signal eliciting root glutathione responses (Haberer et al. 2008). In any case, our data provide no clear evidence that changes in root antioxidants precede above-ground responses, nor that root antioxidant systems responded directly to decreasing SWC.

\section{Conclusions}

- Response of A. aneura was consistent with anisohydric behaviour which allowed stomata to remain open and continue to fix carbon at earlier stages of the experiment.

- Photodamage to PSII (as judged by decreases in maximum quantum efficiency of PSII) developed in both Acacia species only right after water was withheld and the second heatwave imposed, and this response was more gradual for $A$. aneura.

- In phyllodes, total glutathione and ascorbic acid pools increased in response to heatwave rather than drought stress alone, for A. melanoxylon already after the first heat episode and $A$. aneura after the second heatwave. In A. melanoxylon, but not $A$. aneura, ascorbic acid concentrations increased in response to the second heatwave in well-watered trees, and decreases in efficiency of open reaction centres in PSII in the light (but not maximum quantum efficiency of PSII) indicated that flexible heat dissipation processes were engaged in A. melanoxylon, but not $A$. aneura, at earlier stages of the experiment.

- In roots, non-enzymatic antioxidants were unresponsive to drought stress but increased glutathione concentrations in response to the second heatwave in both Acacia species. The response of total ascorbic acid was weaker, and significant changes were observed only in the ratio of reduced to total ascorbic acid.

Acknowledgments AW-K would like to acknowledge staff and students at the Department of Forest and Ecosystem Science of the University of Melbourne. Special thanks to Raymond Dempsey, Markus Loew, Sabine Tausz-Posch and Nina Hinko-Najera for advice during work. We thank reviewers and the editor for many constructive suggestions that improved the manuscript.

Funding AW-K is supported by a Melbourne International Research Scholarship and a Melbourne International Fee Remission Scholarship.

\section{References}

Allen CD, Macalady AK, Chenchouni H, Bachelet D, McDowell N, Vennetier M, Kitzberger T, Rigling A, Breshears DD, Hogg EHT, Gonzalez P, Fensham R, Zhang Z, Castro J, Demidova N, Lim J-H, Allard G, Running SW, Semerci A, Cobb N (2010) A global overview of drought and heat-induced tree mortality reveals emerging climate change risks for forests. Forest Ecol Manag 259:660-684
ANBG (2010) Australian National Botanic Gardens, Growing Native Plants, Australian Government, Canberra, http://www.anbg.gov.au/ gnp/. Accessed 15 July 2013

Asada K (2006) Production and scavenging of reactive oxygen species in chloroplasts and their functions. Plant Physiol 141:391-396

Brodribb TJ, Cochard H (2008) Hydraulic failure defines the recovery and point of death in water-stressed conifers. Plant Physiol 149:575584

Chaves MM, Flexas J, Pinheiro C (2009) Photosynthesis under drought and salt stress: regulation mechanisms from whole plant to cell. Ann Bot London 103:551-560

Costermans L (ed) (2006) Trees of Victoria and adjoining areas. Costermans Publishing, Frankston

Demmig-Adams B, Cohu CM, Muller O, Adams WW (2012) Modulation of photosynthetic energy conversion efficiency in nature: from seconds to seasons. Photosynth Res 113:75-88

Foyer CH, Noctor G (2009) Redox regulation in photosynthetic organisms: signaling, acclimation, and practical implications. Antioxid Redox Signal 11:861-905

Foyer $\mathrm{CH}$, Noctor G (2011) Ascorbate and glutathione: the heart of the redox hub. Plant Physiol 155:2-18

Foyer CH, Neukermans J, Queval G, Noctor G, Harbinson J (2012) Photosynthetic control of electron transport and the regulation of gene expression. J Exp Bot 63:1637-1661

Haberer K, Herbinger K, Alexou M, Rennenberg H, Tausz M (2008) Effects of drought and canopy ozone exposure on antioxidants in fine roots of mature European beech (Fagus sylvatica). Tree Physiol 28:713-719

Hennessy K, Fawcett R, Kirono D, Mpelasoka F, Jones D, Bathols J, Whetton P, Smith MS, Howden M, Mitchell C, Plummer N (2008) An assessment of the impact of climate change on the nature and frequency of exceptional climatic events. CSIRO, Bureau of Meteorology

Herschbach C, Scheerer U, Rennenberg H (2009) Redox states of glutathione and ascorbate in root tips of poplar (Populus tremula $\mathrm{x}$ $P$. alba) depend on phloem transport from the shoot to the roots. $\mathrm{J}$ Exp Bot 61:1065-1074

IPCC, R (2013) Climate change 2013: the physical science basis. Working Group I Contribution to the Fifth Assessment Report of the Intergovernmental Panel on Climate Change. T. F. Stocker, D. Qin, G. K. Plattner et al. New York, Intergovernmental Panel on Climate Change

Johnson DM, McCulloh KA, Reinhardt K (2011) The earliest stages of tree growth: development, physiology and impacts of microclimate. In: Meinzer FC, Lachenbruch B, Dawson TE (eds) Size- and agerelated changes in tree structure and function. Springer Science+ Business Media B.V, Dordrecht

Kim C, Apel K (2013) Singlet oxygen-mediated signaling in plants: moving from flu to wild type reveals an increasing complexity. Photosynth Res 116:455-464

Knörzer OC, Burner J, Boger P (1996) Alterations in the antioxidative system of suspension-cultured soybean cells (Glycine max) induced by oxidative stress. Physiol Plant 97:388-396

Koenker R, Portnoy S, Ng PT, Zeileis A, Grosjean P, Ripley BD (2013) Quantile regression. http://CRAN.R-project.org/package=quantreg: $1-90$

Kubiske ME, Abrams MD (1994) Ecophysiological analysis of woody species in contrasting temperate communities during wet and dry years. Oecologia 98:303-312

Maxwell K, Johnson GN (2000) Chlorophyll fluorescence - a practical guide. J Exp Bot 51:659-668

McDowell N, Pockman WT, Allen CD, Breshears DD, Cobb N, Kolb T, Plaut J, Sperry J, West A, Williams DG, Yepez EA (2008) Mechanisms of plant survival and mortality during drought: why do some plants survive while others succumb to drought? New Phytol 178:719-739 
Munné-Bosch S (2005) The role of $\alpha$-tocopherol in plant stress tolerance. J Plant Physiol 162:743-748

Noctor G, Mhamdi A, Chaouch S, Han YI, Neukermans J, MarquezGarcia B, Queval G, Foyer CH (2012) Glutathione in plants: an integrated overview. Plant Cell Environ 35:454-484

Noctor G, Mhamdi A, Foyer CH (2014) The roles of reactive oxygen metabolism in drought: not so cut and dried. Plant Physiol 164: $1636-1648$

O'Grady AP, Cook PG, Eamus D, Duguid A, Wischusen JDH, Fass T, Worldege D (2009) Convergence of tree water use within an aridzone woodland. Oecologia 160:643-655

Potters G, Gara LD, Asard H, Horemans N (2002) Ascorbate and glutathione: guardians of the cell cycle, partners in crime? Plant Physiol Biochem 40:537-548

Rahantaniaina MS, Tuzet A, Mhamdi A, Noctor G (2013) Missing links in understanding redox signaling via thiol/disulfide modulation: how is glutathione oxidized in plants? Front Plant Sci 4:477

Rennenberg H, Loreto F, Polle A, Brilli F, Fares S, Beniwal RS, Gessler A (2006) Physiological responses of forest trees to heat and drought. Plant Biol 8:556-571

Sgherri CLM, Loggini B, Puligaa S, Navari-Izzo F (1994) Antioxidant system in Sporobolus stapfianus: changes in response to desiccation and rehydration. Phytochemistry 35:561-565

Sharkey TD (2005) Effects of moderate heat stress on photosynthesis: importance of thylakoid reactions, rubisco deactivation, reactive oxygen species, and thermotolerance provided by isoprene. Plant Cell Environ 28:269-277
Sharkey TD, Zhang R (2010) High temperature effects on electron and proton circuits of photosynthesis. J Integr Plant Biol 52: $712-722$

Shvaleva AL, Silva FCE, Breia E, Jouve L, Hausman JF, Almeida MH, Maroco JP, Rodrigues ML, Pereira JS, Chaves MM (2005) Metabolic responses to water deficit in two Eucalyptus globulus clones with contrasting drought sensitivity. Tree Physiol 26:239248

Smirnoff N (1993) The role of active oxygen in the response of plants to water deficit and desiccation. New Phytol 125:27-58

Smirnoff N (2011) Vitamin C: the metabolism and functions of ascorbic acid in plants. Adv Bot Res 59:107-177

SpeciesBank (2014) Australian Biological Resources Study, Canberra. http://www.environment.gov.au/biodiversity/abrs/online-resources/ species-bank/index.html. Accessed 03 April 2014

Tausz M, Šircelj H, Grill D (2004) The glutathione system as a stress marker in plant ecophysiology: is a stress-response concept valid? J Exp Bot 55:1955-1962

Teskey R, Wertin T, Bauweraerts I, Ameye M, McGuire MA, Steppe K (2014) Responses of tree species to heat waves and extreme heat events. Plant Cell Environ. doi:10.1111/pce.12417

Wujeska A, Bossinger G, Tausz M (2013) Responses of foliar antioxidative and photoprotective defence systems of trees to drought: a metaanalysis. Tree Physiol 33:1018-1029

Zhao J, Davis LC, Verpoorte R (2005) Elicitor signal transduction leading to production of plant secondary metabolites. Biotechnol Adv 23: 283-333 